\title{
Optimization of 3D controlled ELM-free state with recovered global confinement for tokamak fusion plasmas
}

SangKyeun Kim ( $\sim$ sk42@princeton.edu )

Princeton University https://orcid.org/0000-0002-0701-8962

Ricardo Shousha

Princeton University

SangHee Hahn

Korea Institute of Fusion Energy

Andrew Nelson

Princeton University

Josiah Wai

Princeton University

SeongMoo Yang

Princeton Plasma Physics Laboratory

Jong-Kyu Park

Princeton Plasma Physics Laboratory

Raffi Nazikian

Princeton Plasma Physics Laboratory

YoungMu Jeon

Korea Institute of Fusion Energy https://orcid.org/0000-0002-7374-3759

YongKyoon In

Ulsan National Institute of Science Technology

Jaehyun Lee

Korea Institute of Fusion Energy

JaeWook Kim

Korea Institute of Fusion Energy

ChanYoung Lee

Seoul National University

Yong-Su Na

Seoul National University https://orcid.org/0000-0001-7270-3846

\section{Egemen Kolemen}

Princeton University https://orcid.org/0000-0003-4212-3247 
Article

Keywords: tokamak fusion plasmas, energy engineering, edge-localized modes (ELMs)

Posted Date: July 26th, 2021

DOl: https://doi.org/10.21203/rs.3.rs-713840/v1

License: (c) (i) This work is licensed under a Creative Commons Attribution 4.0 International License. Read Full License 
Optimization of 3D controlled ELM-free state with recovered global confinement for tokamak fusion plasmas

S.K.Kim, ${ }^{1}$ R.Shousha, ${ }^{1}$ S.H.Hahn, ${ }^{2}$ A.O.Nelson, ${ }^{1}$ J.Wai, ${ }^{1}$ S.M.Yang, ${ }^{3}$ J.-K.Park, ${ }^{3}$

R.Nazikian, ${ }^{3}$ Y.M.Jeon, ${ }^{2}$ Y.In, ${ }^{4}$ J.H.Lee, ${ }^{2}$ J.Kim, ${ }^{2}$ C.Y. Lee, ${ }^{5}$ Y.-S. Na, ${ }^{5}$ and

E.Kolemen ${ }^{1,3}$

1) Princeton University

2) Korea Institute of Fusion Energy

3) Princeton Plasma Physics Laboratory

4) Ulsan National Institute of Science Technology

${ }^{5)}$ Seoul National University

(Dated: 14 July 2021)

Mitigation of deleterious heat flux from edge-localized modes (ELMs) on fusion reactors is often attempted with 3D perturbations of the confining magnetic fields. However, the established technique of resonant magnetic perturbations (RMPs) also degrades plasma performance, complicating implementation on future fusion reactors. In this paper, we introduce an adaptive real-time control scheme as a viable approach to simultaneously achieve both ELM-free states and recovered high-confinement $\left(\beta_{\mathrm{N}} \sim 1.91, \beta_{\mathrm{p}} \sim 1.53\right.$, and $H_{98} \sim 0.9$ ), demonstrating successful handling of a volatile complex system through adaptive measures. We show that, by exploiting a salient hysteresis process to adaptively minimize the RMP strength, stable ELM suppression can be achieved while actively encouraging confinement recovery. This is made possible by a self-organized transport response in the plasma edge which reinforces the confinement improvement through a widening of the ion pedestal and promotes control stability, in contrast to the deteriorating effect on performance observed in standard RMP experiments. These results establish the real-time approach as an up-and-coming solution towards an optimized ELM-free state, which is an important step for the operation of ITER and reactor-grade tokamak plasmas. Notably, the real-time adaptive control scheme introduced here provides a path towards economic fusion reactors by maximizing the fusion gain while minimizing damage to machine components. 
For any fusion energy source to be viable in the global marketplace, it must be able to produce large amounts of electricity without incurring significant damage on the machine. The leading approach towards this goal is a tokamak run robustly in the high confinement mode (H-mode), which is characterized by a narrow edge transport barrier responsible for significantly elevated plasma pressures throughout the device ${ }^{1}$. This "pedestal" not only enhances performance in the core region but also increases the non-inductive current, improving the fusion economy by reducing the external heating and recirculating power required for steady-state operation. Because of these advantages, the ITER baseline scenario ${ }^{2}$ plans to utilize $\mathrm{H}$-mode plasmas to demonstrate ignition in a tokamak for the first time. However, H-mode also presents serious risks to reactor operation, most prominently through the creation of dangerous edge instabilities called edge localized modes $(\mathrm{ELMs})^{3}$. These rapid relaxations of the pedestal density and temperature result in intense transient heat fluxes on the reactor walls, leading to undesired material erosion and surface melting which will not be acceptable in a reactor scenario ${ }^{4,5}$. Therefore, to retain the tokamak design as a viable option for fusion reactors, it is critical that we develop methods to routinely suppress ELM events without degrading the plasma performance.

One of the most effective methods to control ELMs is to apply resonant magnetic perturbations (RMPs) using 3D coils ${ }^{6-9}$. RMPs suppress ELMs by causing additional transport ${ }^{10-23}$ in the pedestal, degrading its height to a point where ELMs are no longer unstable ${ }^{24-26}$. However, this inevitably comes at the considerable expense of global confinement deterioration, decreased access to high-performance plasma regimes and thus depleted economic prospects. This degradation tends to be greater with a lower toroidal wave number $(n)$ of RMP. Even so, the use of low- $n$ configurations will be important at the reactor level due to the strong decay of external fields in the thick shielding between the plasma and field coils. Undoubtedly, the compatibility of RMP ELM suppression with high confinement operation requires urgent exploration.

In this context, we report on an adaptive RMP scheme capable of maximizing plasma performance while maintaining robust ELM suppression. With this new technique, up to $\sim 70 \%$ of the RMP-induced performance degradation can be quickly recovered, returning the plasma to a highpower state suitable for future reactors. By exploiting a salient hysteresis process on the KSTAR tokamak ${ }^{27}$, we find that RMP-induced transport does not just produce a negative influence on confinement (as is typically assumed) but instead also opens up a pathway to strong recovery of plasma performance that is accessible to a highly-optimized controller. This leads to the concurrent establishment of high confinement plasmas and sustained ELM suppression at normalized 

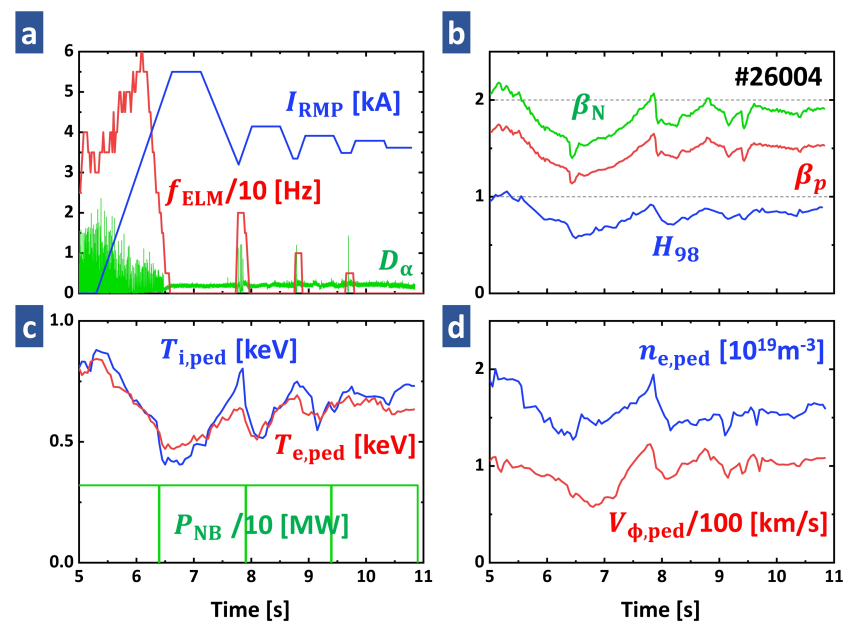

FIG. 1. Plasma parameters for an ELM suppression discharge (\#26004) with adaptive RMP control. a RMP coil current (blue), $D_{\alpha}$ emission (green) near outer divertor target, and detected ELM frequency (red). b $H_{98}$ (blue), $\beta_{\mathrm{N}}$ (green), and $\beta_{\mathrm{p}}$ (red). $\mathbf{c}$ Pedestal height of ion (red), electron (blue) temperature, and NBI heating power (green). d Pedestal height of electron density (blue) and toroidal rotation of carbon (6+) impurity (red).

performance close to the ITER-baseline level, reaching $\beta_{\mathrm{N}} \sim 1.91, \beta_{\mathrm{p}} \sim 1.53$, and $H_{98} \sim 0.9$. Here, $\beta_{\mathrm{N}}=\frac{a B_{T}}{I_{p}} \frac{p}{B^{2} / 2 \mu_{0}}$ is the normalized beta, $\beta_{\mathrm{p}}=\frac{p}{B_{p}^{2} / 2 \mu_{0}}$ is the poloidal beta, and $H_{98}=\tau_{\exp } / \tau_{98}$ is the thermal energy confinement quality compared to the standard H-mode plasmas, where $p$ is the averaged plasma pressure, $a$ is the minor radius, $I_{p}$ is the total plasma, $B_{T}$ is the toroidal magnetic field, $B_{\mathrm{p}}$ is the poloidal magnetic field, $B$ is the total magnetic field, $\tau_{\exp }$ is the experimental thermal energy confinement time, and $\tau_{98}$ is the empirically derived confinement time using standard $\mathrm{H}$-mode database ${ }^{28}$. Since $H_{98}$ enters to the power of 3.23 in determining the fusion gain $Q_{\text {fus }}{ }^{29}$, where $Q_{\text {fus }}$ is the ratio between produced fusion energy over input, the strong recovery of $H_{98}$ demonstrated in this work allows a substantial reduction of fusion cost, establishing a means with which RMPs can be used for ELM suppression to enable commercial-grade fusion devices.

\section{RESULTS}

Optimized pedestal using adaptive control. Figure.1 shows an example of H-mode plasma with fully suppressed ELMs via adaptive feedback RMP amplitude control. In this discharge, a hysteresis effect is utilized where ELM suppression can be maintained over long periods with a lower RMP strength than initially required for access to the ELM suppression regime ${ }^{17}$. Because 
reduction of the RMP amplitude leads to an increased pressure pedestal height, this enables global confinement recovery in an ELM-free state ${ }^{30}$ by adjusting RMP levels. To avoid ELMs while maximizing the confinement, we use a preset low $n=1 \mathrm{RMP}_{\text {waveform }}^{8}$ and apply real-time feedback to control its amplitude. During the plasma current flattop before applying RMP, with $I_{p}=0.51 \mathrm{MA}$ and $\sim 3 \mathrm{MW}$ of neutral beam injection heating, $\beta_{\mathrm{N}} \sim 2.13, \beta_{\mathrm{p}} \sim 1.71$, and $H_{98} \sim$ 1.03, close to the targets of the proposed ITER baseline scenario. In this discharge, the plasma edge safety factor $q_{95} \sim 5$, which is higher than the target value of $q_{95} \sim 3$. Here, $q_{95}$ is defined as the pitch of the magnetic field line in the edge where the normalized poloidal flux $\left(\psi_{\mathrm{N}}\right)$ is 95\%. However, after achieving the first stable ELM suppression through traditional means (7.1 s), the plasma performance significantly decreases to $\beta_{\mathrm{N}} \sim 1.62, \beta_{\mathrm{p}} \sim 1.30$, and $H_{98} \sim 0.68$. The $30 \%$ reduction in overall confinement by RMP mainly comes from degradation in density and temperature pedestal, as shown in Fig.1c, d. Such extensive confinement and $H_{98}$ degradation is a well-known general trend in low-n RMP experiments ${ }^{31-33}$ and will not be acceptable in a future fusion reactor because this leads to a significant increase in fusion cost.

After this initial degradation, the real-time adaptive ELM control scheme starts to recover the original performance before RMPs were introduced while maintaining stable ELM suppression. The controller leverages the $D_{\alpha}$ emission signal near the outer divertor target to calculate the frequency of ELMs $\left(f_{\mathrm{ELM}}\right)^{34}$. To achieve ELM suppression, the RMP amplitude (or coil current, $I_{\mathrm{RMP}}$ ) is raised until $f_{\mathrm{ELM}}$ decreases to 0, i.e., ELM suppression. Then, during the resulting ELM-free period, the controller lowers the RMP strength to raise the pedestal height until ELMs reappear, at which point the control again starts to ramp up the RMP amplitude until suppression is recovered (Fig.1a). In the experiment presented in Fig.1, there are $0.5 \mathrm{~s}$ of RMP flattop intervals between the RMP-ramp up and down phase to achieve saturated RMP response. Throughout this process, we adjust the lower bound of $I_{\text {RMP }}$ to match the value where the most recent ELM returns. This adaptive constraint reduces the likelihood of ELM suppression loss and control oscillation. The feedback system leads the plasma to a converged operating point that optimizes both ELMfree operation and confinement, recovering most of the performance lost in the initial application of RMP.

In the selected discharge, this adaptive ELM control scheme achieves a stable ELM-free phase at $10.5 \mathrm{~s}$ with improved global confinement, as shown in Fig.1b. Although a few ELMs occur before convergence, the controller successfully reaches a stable operating point with minimized ELMy periods. In the final state, the plasma performance shows $\beta_{\mathrm{N}} \sim 1.91, \beta_{\mathrm{p}} \sim 1.53$, and 

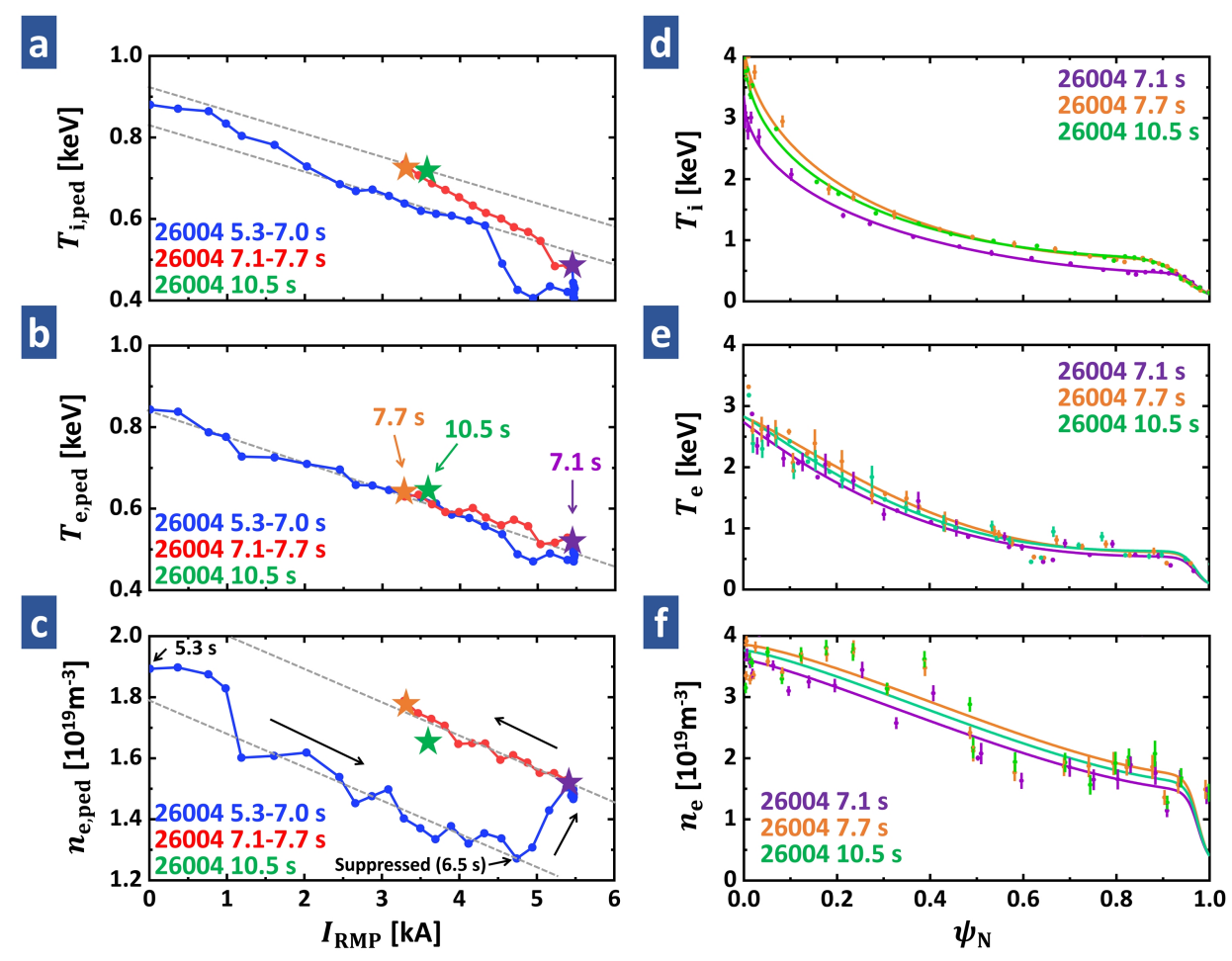

FIG. 2. Pedestal height (left) and core plasma profiles (right) for RMP ramp-up (5.3-7.1 s, blue), down (7.1-7.7 s, red), first saturated ELM-suppression (7.1 s, purple), first optimized suppression (7.7 s, orange), and finally optimized suppression (10.5 s, green). a-c Pedestal height of ion, electron temperature and electron density. d-f Core ion, electron temperature, and electron density with statistical error bars. Ion temperature is measured by a charge-exchange recombination system for carbon (6+) impurities. Electron temperature is measured by the Thomson Scattering and Electron cyclotron emission system. Electron density is measured by the Thomson Scattering and Two-color interferometry system.

$H_{98} \sim 0.9$, recovering up to $68 \%$ of the original confinement degradation. Such increase in $H_{98}$ is especially important as this leads to the $60 \%$ recovery in $Q_{\text {fus }}$ degradation, thus emphasizing the performance of adaptive control. The enhanced confinement quality occurs with the recovery of both the temperature and density pedestals. As can be seen in Fig.1c, d, all pedestals are significantly improved from the first ELM suppression phase. For example, electron $\left(T_{\mathrm{e}, \mathrm{ped}}\right)$ and ion $\left(T_{\mathrm{i}, \text { ped }}\right)$ temperature pedestals increase by $22 \%$ and $50 \%$, respectively. In addition, the electron density pedestal $\left(n_{\text {e,ped }}\right)$ is also recovered by $10 \%$ at the same time. Interestingly, $H_{98} \sim 0.9$ at $10.5 \mathrm{~s}$ is much larger than $H_{98} \sim 0.75$ at $6.2 \mathrm{~s}$, even with the same $I_{\mathrm{RMP}}=3.6 \mathrm{kA}$. This indicates that the confinement recovery by adaptive approach is not solely attributable to decreased $I_{\mathrm{RMP}}$, but rather that another contributor leads the plasma to a reinforced high-confinement state. 
We note that the ion temperature pedestal exhibits significant recovery compared to the other channels. This is mainly due to the rapid and significant increase of ion pedestal height by decreasing RMP strength. The traces of pedestal height versus $I_{\text {RMP }}$ before the first ELM reappearance (5.3-7.7s) reveal this trend, as shown in Fig.2a-c. $n_{\mathrm{e}, \text { ped }}$ and $T_{\mathrm{e}, \mathrm{ped}}$ have a similar dependence on $I_{\mathrm{RMP}}$ during the pedestal degradation (5.3-6.5s) and recovery $(7.1-7.7 \mathrm{~s})$ phases, showing $\frac{\Delta n_{\text {e,ped }}}{\Delta I_{\text {RMP }}} \sim-10^{15} / \mathrm{m}^{3} \mathrm{~A}$ and $\frac{\Delta T_{\text {eped }}}{\Delta I_{\text {RMP }}} \sim-0.06 \mathrm{eV} / \mathrm{A}$. However, $T_{\mathrm{i} \text {,ped }}$ in the recovery phase shows a $50 \%$ larger response of $-0.09 \mathrm{eV} / \mathrm{A}$ compared to the degradation phase, $-0.06 \mathrm{eV} / \mathrm{A}$. The difference of responses in these phases leads to the faster and larger recovery of the ion pedestal. As shown in Fig.2d-f, all radial profiles in the core plasma are almost identical during the recovery phase. Therefore, the improved confinement by decreasing RMP strength results from increased $n_{\mathrm{e}, \mathrm{ped}}, T_{\mathrm{e}, \text { ped }}$, and $T_{\mathrm{i}, \text { ped }}$, with the last one dominant. In particular, $\sim 67 \%$ of improvement comes from the ion pedestal, and this is responsible for reinforced recovery by adaptive control. The large growth of $T_{\mathrm{i}, \text { ped }}$ is mainly due to the simultaneously increased upper limit of $T_{\mathrm{i} \text {,ped }}$ before the loss of ELM suppression and its enhanced response to the RMP strength. In addition, $n_{\mathrm{e}, \text { ped }}$ shows a large increase near $I_{\mathrm{RMP}} \sim 5 \mathrm{kA}$ (Fig.2c), which can be attributed to reduced particle pumping from ELMs. This occurs before $7 \mathrm{~s}$ and does not directly contribute to confinement recovery beginning at $7.1 \mathrm{~s}$. However, it still strengthens the confinement recovery with increasing $T_{\mathrm{i} \text {,ped }}$.

Advantages of the adaptive ELM control for achieving safe ELM suppression. In standard H-mode discharges, strong RMPs are favorable for entering the ELM suppression but also raises the possibility of dangerous plasma destabilization. Too large of an RMP field in the core plasma normally leads to a locking of plasma rotation and invokes a disastrous core instability called a disruption, as seen in Fig.3a. Core locking (or disruptions) terminate the plasma and forms transient heat fluxes on the tokamak walls which are even more severe than ELMs. Unfortunately, plasma disruption is easier with low-n RMPs. Therefore it is vital to maintain the RMP strength between the thresholds of ELM suppression and disruption. To complicate this process, these thresholds change in time with various plasma parameters and are often hard to theoretically predict. The database $^{33}$ for $n=1$ RMP ELM suppression in KSTAR reveals broadly scattered experimental thresholds showing $1 \sim 2 \mathrm{kA}$ variations, and empirical prediction is also challenging due to their sensitivity to plasma parameters. For these reasons, in the present experiments, a series of discharges are used to find safe RMP strength for ELM suppression. This approach will not be applicable in a fusion reactor, where a single disruption can result in the termination of machine 


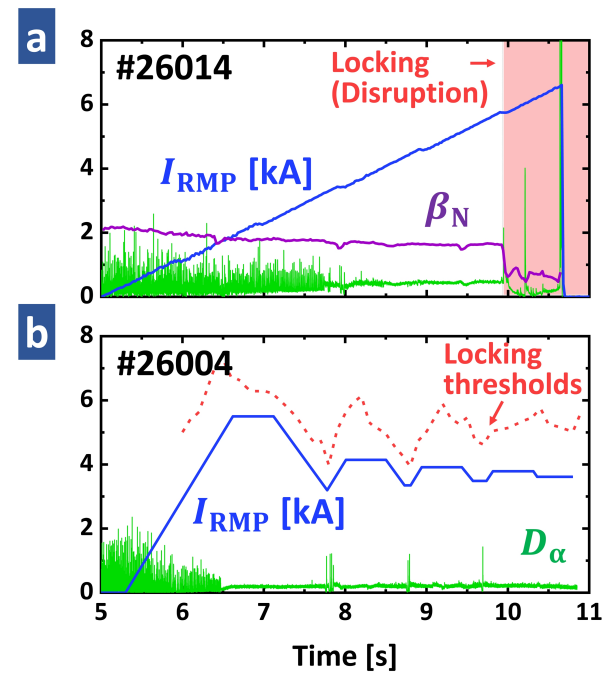

FIG. 3. Plasma parameters for a RMP-induced disruption and suppression discharge with $n=1$ RMP in KSTAR. a RMP coil current (blue), $D_{\alpha}$ emission (green), and $\beta_{\mathrm{N}}$ (purple) of discharge \#26014. Onset of locking (disruption) is marked as a red region. b RMP coil current (blue) and $D_{\alpha}$ emission (green) of discharge \#26004. The disruption thresholds in $I_{\mathrm{RMP}}$ is marked as a red dotted line.

life.

Notably, the adaptive approach lowers the RMP strength after entering the ELM-free state and maintains it near the levels for marginally stable ELM suppression. This automatically avoids touching the disruptive limits. As shown in Fig.3b, the RMP strength stays safely below the disruption threshold throughout the example discharge, highlighting the advantages of this adaptive scheme for achieving stable ELM suppression. Here, the disruption thresholds are predicted from adjacent RMP-disruption experiments and ideal RMP response calculations. Although adaptive RMP control will be ineffective if only a small margin exists between the thresholds for suppression and disruption, it still reduces the necessity of extensive optimization of the RMP geometry for locking avoidance, which often comes at the expense of other important parameters or operational degrees of freedom.

\section{Improved ELM stability and ion pedestal response by RMP-induced transports. Instead of} causing only degradation of pedestal, RMP-induced pedestal transport facilitates the improvement of the $T_{i, p e d}$ limit and its response to the RMP strength by broadening the ion-pedestal. RMPinduced transport on the ion pedestal can be found from the analysis of the ion pedestal profiles in detail. Fig.4a, b illustrate ion pedestal and $E \times B$ flow profiles for five times between 5.3 and 7.7 


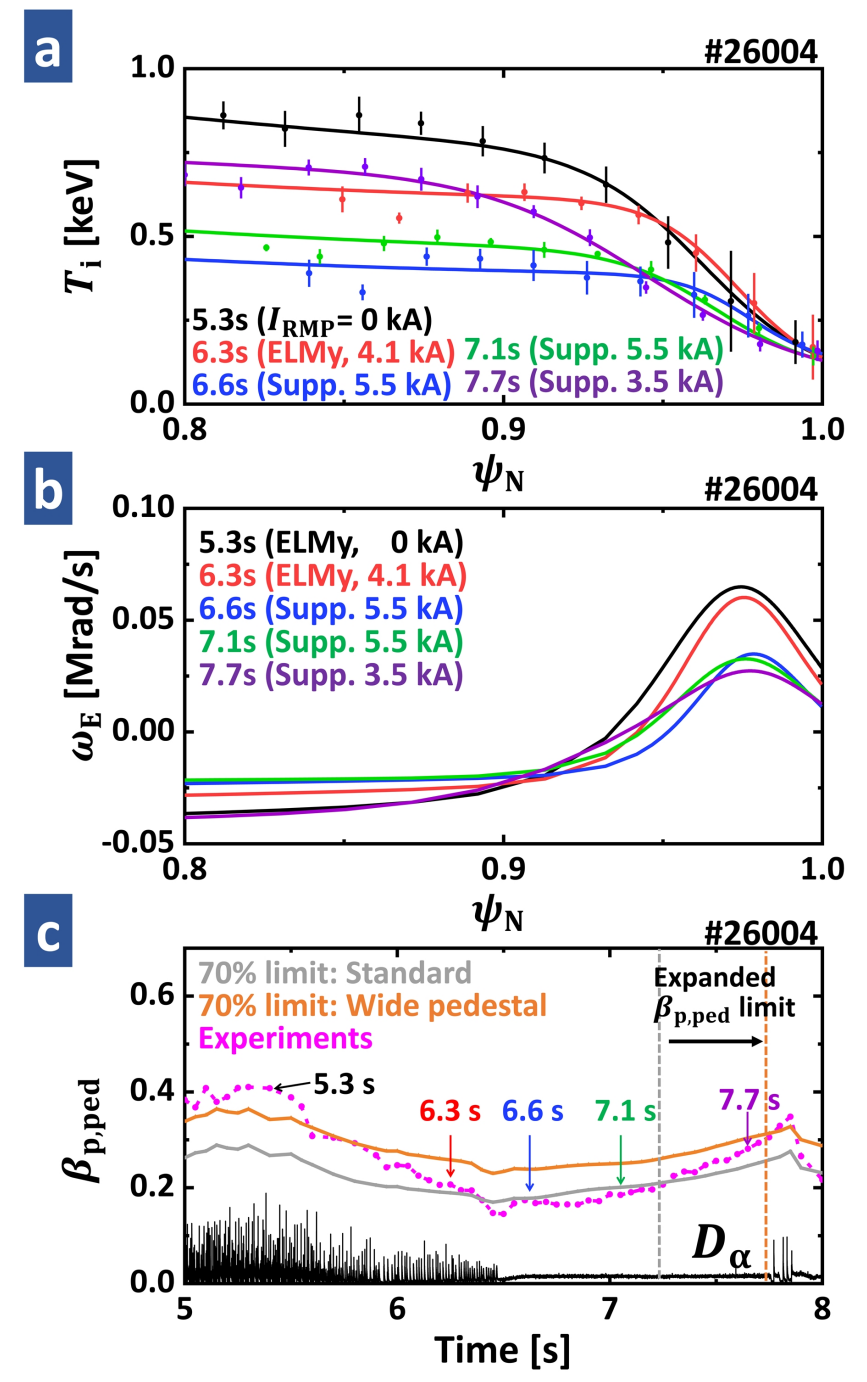

FIG. 4. Time traces of pedestal profiles and stability limits during adaptive ELM control (\#26004). a Ion pedestal profiles with statistical error bars are shown for five different time slices. b ExB flow profiles $\left(\omega_{\mathrm{E}}\right)$ at pedestal are shown for five different time slices. c $70 \%$ of ELM stability limit for $\beta_{\mathrm{p}, \text { ped }}$ with (orange) and without (gray) wide ion pedestal, calculated from EPED code. Experimentally measured $\beta_{\text {p,ped }}$ (magenta) and $D_{\alpha}$ emission (black) are also shown. The dotted lines show $\beta_{\mathrm{p} \text {,ped }}$ limits during ELM-free state imposed by pedestal stability with (gray) and without (orange) wide ion pedestal.

s. Before ELM suppression (5.3-6.3 s), $T_{\mathrm{i} \text {,ped }}$ decreases with $I_{\mathrm{RMP}}$, while the pedestal gradient is well sustained (or even slightly increased). After ELM suppression ( $>6.5 \mathrm{~s}$ ), however, the pedestal stiffness starts to change. The transition from 6.6 to $7.1 \mathrm{~s}$ shows broadening of the ion pedestal and decreasing of its gradient. This widening is maintained in the pedestal recovery phase up to 7.7 s. The decrease in pedestal height and gradient are both due to RMP-induced transport. However, 
the rapid broadening of the ion pedestal after ELM suppression indicates that its gradient is not governed by the transport affecting the pedestal height but instead by an "additional" transport source that occurs in the ELM suppression phase.

The change in ion pedestal width improves the ELM stability. In theory, pedestal pressure $\left(P_{\text {ped }}\right)$ or pedestal poloidal beta $\left(\beta_{\mathrm{p}, \text { ped }}=\frac{P_{\text {ped }}}{B_{p}^{2} / 2 \mu_{0}}\right)$ should stay under the stability limit to avoid the reappearance of ELM crashes. Stability analysis confirms that $\beta_{\mathrm{p}, \mathrm{ped}}$ stays below $70 \%$ of the stability limit during the ELM suppression phase. This stability limit is known to improve with increased pedestal width ${ }^{35}$. Therefore, widened pressure pedestal via ion-pedestal broadening allows for higher $\beta_{\mathrm{p} \text {,ped }}$ during the ELM-free phase. Numerical analysis reveals that the $\beta_{\mathrm{p} \text {,ped }}$ limit increases by $53 \%$ due to ion pedestal broadening. This change is presented in Fig.4c. With the expansion of the $\beta_{\mathrm{p}, \mathrm{ped}}$ limit illustrated as dotted lines, $\beta_{\mathrm{p}, \mathrm{ped}}$ can further increase from 0.2 (gray dotted line) to 0.31 (orange dotted line). This enhanced $\beta_{\mathrm{p}, \text { ped }}$ limit allows access to higher $T_{\mathrm{i}, \mathrm{ped}}$ in the ELM suppression phase.

The broader ion-pedestal also can lead a larger response of $T_{\mathrm{i} \text {,ped }}$ on RMP strength. Inspired from (Hu et al. 2020) ${ }^{36}$, the change of pedestal height $\left(\Delta T_{\text {ped }}\right)$ by $\Delta I_{\text {RMP }}$ can be described as Eq.1,

$$
\frac{\Delta T_{\text {ped }}}{\Delta I_{\mathrm{RMP}}} \approx \nabla T_{\text {ped }} \sum_{m \geq q_{\mathrm{ped}}} \frac{\partial W_{\mathrm{m}, \mathrm{n}}}{\partial I_{\mathrm{RMP}}},
$$

where $W_{\mathrm{m}, \mathrm{n}}$ and $\nabla T_{\text {ped }}$ are the $(m, n)$ island width and pedestal gradient, respectively. $q_{\text {ped }}$ is an edge safety factor on the pedestal top. This expression is based on the concept where $\Delta T_{\text {ped }}$ is the accumulation of profile flattening by the islands in the pedestal region. We note that constant $\nabla T_{\text {ped }}$ over the pedestal region is assumed to make interpretation easier. This expression addresses that pedestal height changes more rapidly with RMP strength as the pedestal gradient grows and $q_{\text {ped }}$ decreases. With the given q profile monotonic, $q_{\text {ped }}$ is reduced by increasing pedestal width. The largely broadened ion pedestal can lead to a stronger response of $T_{\mathrm{i} \text {,ped }}$ despite the decrease of ion pedestal gradient. In addition, ion pedestal is known to be heavily influenced by neoclassical transport ${ }^{15,37,38}$. Here, neoclassical heat flux by RMPs is roughly proportional to $I_{\mathrm{RMP}}^{2}$, and it increases more rapidly with the smaller radial electric field and its gradient ${ }^{39,40}$. Because a wide ion pedestal reduces the electric field ${ }^{19,41}$ at the pedestal (Fig.4b), this correlation also contributes to improving the response of $T_{\mathrm{i}, \mathrm{ped}}$.

On the other hand, the responses of $n_{\mathrm{e}, \text { ped }}$ and $T_{\mathrm{e} \text {,ped }}$ to RMP strength are almost identical whether or not the ELMs are fully suppressed. This means that additional RMP-induced transport in the ELM-free phase has a smaller effect on the electron density and temperature pedestal gra- 


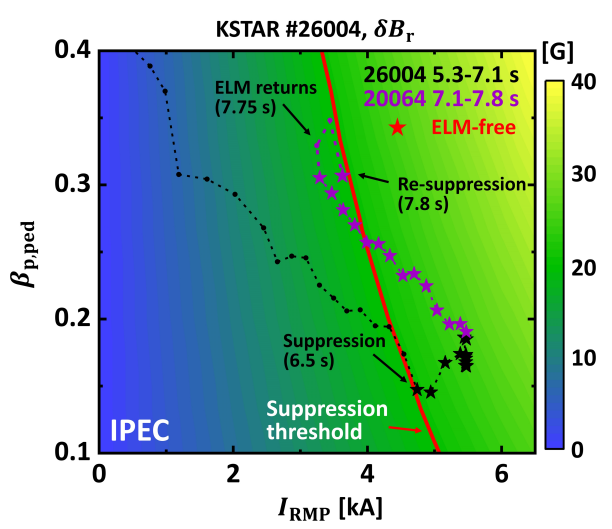

FIG. 5. The pressure pedestal height versus RMP strength during adaptive ELM control (\#26004). The time traces of $\beta_{\mathrm{p} \text {,ped }}$ in \#26004 discharge for 5.3-7.1 s (black) and 7.1-7.8 s (purple) with varying $I_{\mathrm{RMP}}$. ELM-free states are marked as star dots. Contours of $\delta B_{\mathrm{r}}$ at pedestal region from ideal response calculation using IPEC are also shown. Experimentally derived $\delta B_{\mathrm{r}}$ threshold for ELM suppression is drawn as a red curve.

dient. Although the electron pedestal width has considerable uncertainty due to limitations in the resolution of edge diagnostics, its value lies between $4-6 \%$ in normalized poloidal flux without showing a considerable widening like ion pedestal, suggesting that additional transport has only a relatively small effect on electron channels.

Advantages of RMP-induced transport and wide ion pedestal in adaptive ELM control. Increased $T_{\mathrm{i} \text {,ped }}$ response by RMP-induced transport leads to an extensive recovery of $T_{\mathrm{i} \text {,ped }}$ during RMP ramp-down and makes an ion pedestal higher than the RMP ramp-up phase (ELMy) even with the same RMP strength. In addition, enhanced pedestal stability allows for larger $T_{i, \text { ped }}$ before the return of ELMs. The synergy between these effects boosts the pedestal recovery and enables adaptive control to maximize the confinement, resulting in a much higher pedestal than during the initial phase of ELM suppression, as shown in Fig.5, which illustrates $\beta_{\mathrm{p} \text {,ped }}$ versus $I_{\mathrm{RMP}}$. The changes to the pedestal from 5.3 to $7.8 \mathrm{~s}$ are shown, and the ELM suppressed states are marked with star points.

Another advantage of RMP-induced transport is that it improves the control stability. Adaptive control can be unstable due to a bifurcation of the plasma state during transitions between ELMy and ELM-free regimes, which causes oscillation of the control system. In particular, it can take a long time or even become impossible for a controller to find the optimal solution because of 

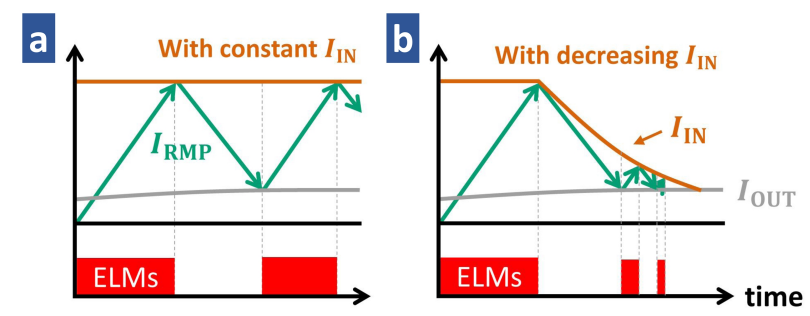

FIG. 6. Schematic diagram of adaptive ELM control using RMPs. Here, RMP threshold for ELM suppression entry $\left(I_{\mathrm{IN}}\right.$, orange) and exit ( $I_{\mathrm{OUT}}$, gray) are drawn. Time trance of $I_{\mathrm{RMP}}$ (green) and onset of ELMs (red box) are also shown. Expected time trace of adaptive ELM control with a constant $I_{\mathrm{IN}}$ and $\mathbf{b}$ decreasing $I_{\mathrm{IN}}$ in time.

the sudden jump in RMP strength required for re-entry $\left(I_{\mathrm{IN}}\right)$ to or exit $\left(I_{\mathrm{OUT}}\right)$ from ELM suppression. The schematic diagram in Fig.6a illustrates how this characteristic will delay the control convergence. In practice, ELM control must be done quickly to minimize damage to the reactor, so an adaptive approach is generally hard to use in such a bifurcating system. However, RMPinduced transport eases these control difficulties by reducing $I_{\mathrm{IN}}$ during adaptive control, as shown in Fig.6b.

It has been shown that the plasma enters the ELM suppression state above a certain $\delta B_{\mathrm{r}}$ threshold ${ }^{42}$, where $\delta B_{\mathrm{r}}$ is the perturbed radial field strength at the pedestal. This threshold $(\sim 20$ G) for the reference discharge is shown as the red contour of Fig.5. Here, $\beta_{\mathrm{p}, \text { ped }}$ amplifies the perturbed field ${ }^{42}$, and the same $\delta B_{\mathrm{r}}$ can be obtained with a smaller $I_{\mathrm{RMP}}$ with larger $\beta_{\mathrm{p} \text {,ped. Because }}$ RMP-induced transport enhances $\beta_{\mathrm{p} \text {,ped }}$ in an ELM-free state, this leads to a lower $I_{\mathrm{IN}}$, making access to the next ELM suppression regime easier. The ELM suppression of $7.8 \mathrm{~s}$ shown in Fig.5 results from reduced $I_{\mathrm{IN}}$ compared to the former one at $6.5 \mathrm{~s}$. Thus, $I_{\mathrm{IN}}$ for each suppression entry changes as $4.9 \rightarrow 3.6 \rightarrow 3.53 \rightarrow 3.5 \mathrm{kA}$, as seen in Fig.1(a), resulting in fast and stable system optimization. This interesting example shows uncommon positive effect ${ }^{43,44}$ of self-organized transport on pedestal confinement.

We note that such an RMP-induced hysteresis shown in Fig5 is not trivial to be produced in the experiment as it conventionally requires a delicate pre-programmed RMP waveform. This leads to difficulties in investigating and exploiting the hysteresis, which is critical to optimize the ELM-free state. In this respect, adaptive RMP control is an effective methodology as it can automatically generate the hysteresis and utilize it. In addition, the adaptive scheme has been successfully operated for more than a hundred confinement times $(\sim 5 \mathrm{~s})$ of KSTAR, and therefore, 
this control is also expected to be applicable to long pulse plasma in ITER.

The origin of broadened ion-pedestal. It is worth pointing out that successful adaptive control in these experiments is mainly due to a broadened ion pedestal during the ELM suppression phase. As shown in Fig.7a, the ion heat diffusivity $\left(\chi_{\mathrm{i}}\right)$ of the pedestal region rapidly increases via additional transport after transitions to the ELM-free state. In addition, the pedestal heat diffusivity does not change much during 7.1-7.7 s, indicating that it is insensitive to the decreasing $I_{\mathrm{RMP}}$. It has been reported that the neoclassical transport effect dominates ion heat transport under RMPs $^{37,38}$. However, this collisional transport strongly depends on the RMP strength. Therefore, the broadened ion pedestal does not seem to be related to the neoclassical process. Here, $\chi_{\mathrm{i}}$ at $\psi_{\mathrm{N}}=0.96$ exceeds neoclassical level $\left(\geq 0.4 \mathrm{~m}^{2} / \mathrm{s}\right)$ in all cases, supporting the existence of additional transport.

Fluctuation measurements on $\operatorname{KSTAR}\left(k_{\mathrm{y}} \rho_{\mathrm{s}}<0.1\right)$ reveal significant edge turbulence triggered by RMPs ${ }^{25,26,45}$ after ELM suppression, where $k_{\mathrm{y}}$ is the bi-normal wave number, $\rho_{\mathrm{s}}=\sqrt{2 m_{i} T_{e}} / e B$ is the hybrid Larmor radius, and $m_{i}$ is deuterium mss. Fig.7c, $d$ illustrate the spectrogram and the coherence strength of $\delta T_{\mathrm{e}}$ and $\delta n_{\mathrm{e}}$ fluctuations at $\psi_{\mathrm{N}} \sim 0.96$. Fig.7e shows the poloidal magnetic field fluctuations $\left(\delta B_{\mathrm{pol}}\right)$ at the inner wall. Here, $\delta T_{\mathrm{e}}$ and $\delta n_{\mathrm{e}}$ have strong coherence over the frequency range of $20-100 \mathrm{kHz}$. The magnetic fluctuations in the $80-400 \mathrm{kHz}$ range are also observed during the same period. As shown in Fig.7f, they show an immediate instigation of turbulence as ELM suppression begins followed by quick saturation within $200 \mathrm{~ms}$. We note that coherence before $6.4 \mathrm{~s}$ comes from ELM noise, and a magnetic signal of $<50 \mathrm{kHz}$ is responsible for core modes. It is noteworthy that the strength of coherent fluctuations remains almost identical during 7.1-7.7 s. Here, the widening of the ion pedestal coincides with the occurrence of edge fluctuations. Furthermore, they are both insensitive to RMP strength. Therefore, these similarities support the claim that the ion pedestal is widened primarily due to increased heat diffusivity by edge turbulence.

Linear gyrokinetic simulations confirms that enhanced edge turbulence may occur in the ELM suppression phase. As shown in Fig.7b, the linear growth rates $\left(\gamma / \gamma_{\mathrm{E}}\right)$ of turbulence mode exceed the onset limit $(>1)$ after the transitions to the ELM-free state. This is mainly due to decreased stabilizing effect from the ExB shearing rate $\left(\gamma_{E}\right)^{46,47}$, which comes from the degraded pressure pedestal (Fig.4b). It turns out that the excited modes are correlated with the ITG/TEM hybrid. Here, the bi-normal wave length $k_{\mathrm{y}} \rho_{\mathrm{s}} \sim 0.3$ and real frequency $\sim 51 \mathrm{kHz}$ of the most unstable 

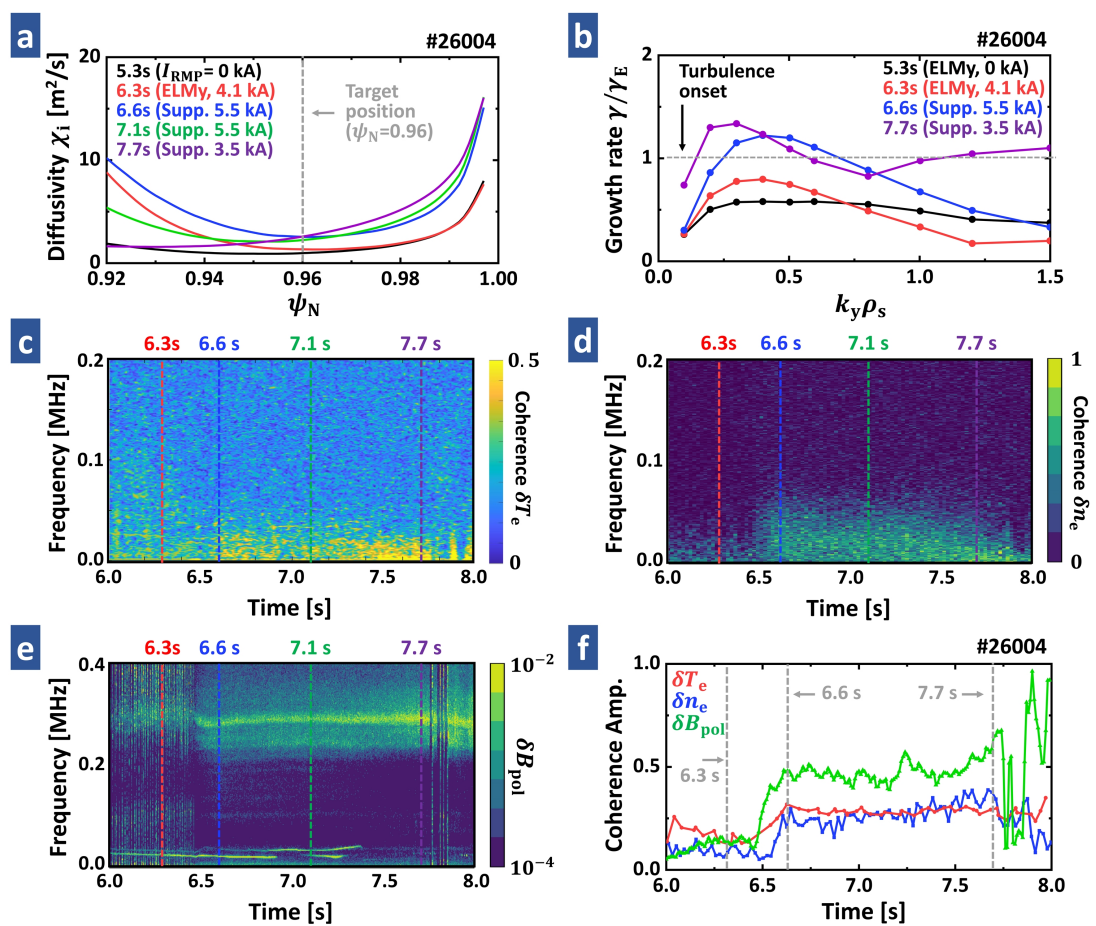

FIG. 7. A broadened ion temperature pedestal by RMP-induced transport during ELM-suppression state. a Radial profiles of ion heat diffusivity $\left(\chi_{\mathrm{i}}\right)$ for five different time slices. b The growth rates of instability calculated from Gyro-kinetic simulation code CGYRO. c Coherence of edge $T_{\mathrm{e}}$ fluctuation from Electron cyclotron emission imaging system. d Coherence of edge $n_{\mathrm{e}}$ fluctuation from Beam emission imaging system. e Measured $\delta B_{\text {pol }}$ fluctuation at inner wall from Mirnov coil. f Time trace of normalized integrated coherence amplitude of $T_{\mathrm{e}}(\mathrm{red}), n_{\mathrm{e}}$ (blue), and $B_{\mathrm{pol}}$ (green) fluctuations over the frequency space.

mode exhibits similar properties to the measured fluctuations of electron channels. The simulation results show that ion thermal diffusion can be increased with these unstable modes, supporting the idea of ion pedestal broadening by turbulence. However, theoretical analysis on RMP-induced turbulence still has many missing pieces. Recent studies have shown that the characteristics of transport in the presence of RMP deviates significantly from linear gyrokinetic calculations, raising the importance of non-linearity ${ }^{48}$ and non-locality ${ }^{49}$. In the future, nonlinear gyrokinetic studies including these effects will shed further light on the accurate description of edge turbulence under RMPs.

The considerable effect of RMP-induced transport on ion heat diffusion might inconsistent with the general trend of other devices ${ }^{16,17,32}$, where such turbulence mainly affects electron channel and has a minor effect on ion transport. Although it is difficult to evaluate the turbulence effect 


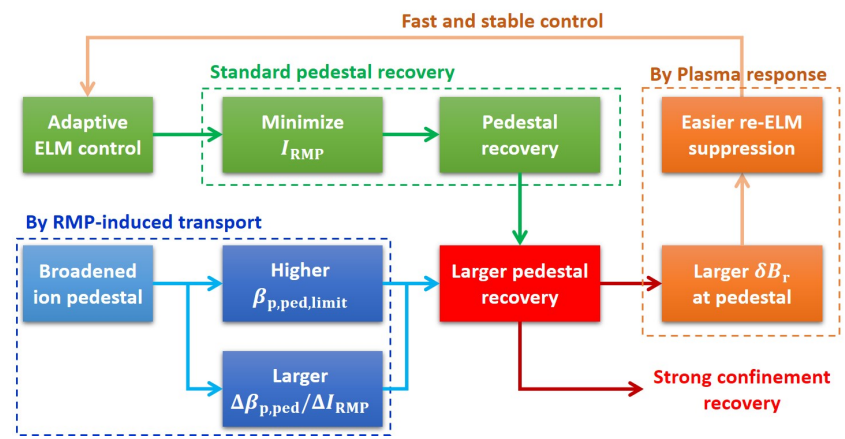

FIG. 8. Schematic diagram of correlation between adaptive ELM control and pedestal recovery. Here, it is noteworthy that the strong recovery of confinement is also attributable to the widened ion pedestal by RMP-induced transport during ELM suppression phase.

on $n_{\mathrm{e}}$ and $T_{\mathrm{e}}$ due to limitations in the diagnostics, we still confirm that there is a clear correlation between edge fluctuation and ion pedestal. Therefore, this observation suggests new possible role of turbulence on the ion pedestal under the low-n(=1) RMP and ELM-free states.

\section{DISCUSSION}

We have achieved successful optimization of a controlled ELM-free state with highly recovered confinement by $\sim 60 \%$, maintaining $\beta_{\mathrm{N}} \sim 1.91, \beta_{\mathrm{p}} \sim 1.53$, and $H_{98} \sim 0.9$, with the original degradation in fusion gain largely recovered. This novel adaptive approach exhibits compatibility between RMP ELM suppression and high confinement. In addition, it provides a reliable strategy to achieve stable ELM-free access by preventing RMP-induced disruption. It is noteworthy that the remarkable recovery of confinement is not solely attributable to adaptive RMP control but also to a widened ion pedestal resulting from RMP-induced transport that promotes pedestal recovery by improving the ion response and ELM stability and facilitates fast, stable, and reinforced control optimization (Fig.8). This feature, which can be correlated to the turbulent process, is a good example of a system that transitions to an optimal state through a self-organized response to adaptive modulation. These results with low $n=1$ RMP confirm that adaptive ELM control is a highly promising approach towards optimizing the ELM-free state, potentially solving one of the most challenging obstacles for viable and economical fusion energy.

However, there are remaining features to be improved for a "complete" adaptive ELM control picture. As shown in Fig.1a, the current approach is based on ELM detection and thereby in- 
evitably faces several ELMs during control. This limitation could be critical at the reactor level, where a single ELM can already be dangerous. Thus, a way to detect the loss of ELM suppression in advance of the ELM re-occurrence is needed. Here, the behavior of edge turbulence suggests the potential solution. The amplitude of magnetic fluctuation during the ELM-free phase shows a rapid decrease $70 \mathrm{~ms}$ before the return of ELMs at $7.75 \mathrm{~s}$ (Fig.7f). Such an abrupt change in magnetic signals is an effective indicator of suppression loss. Therefore, this property can be utilized in real-time to entirely avoid the return of ELM to achieve truly ELM-free optimization.

Previous work has shown that the effectiveness of RMP ELM suppression can be enhanced by physics model-based 3D geometric optimization ${ }^{50}$. Since this adaptive ELM control scheme maximizes the plasma performance for a given scenario, any additional improvements from external forces will be augmented by the adaptive scheme. This makes the adaptive approach a prime candidate to fully exploit existing physics models for RMP ELM suppression. Future integration of these features will lead to broader operational freedom and higher confinement recovery, as well as the development of advanced ELM control techniques for ITER and future tokamaks.

\section{METHODS}

KSTAR tokamak. The KSTAR tokamak is the largest magnetic fusion devices in Republic of Korea, supported by the Korea Institute of Fusion Energy (KFE) and the Government funds. It has the plasma major radius $R_{0}=1.8 \mathrm{~m}$, minor radius $a_{0}=0.45 \mathrm{~m}$, and the toroidal magnetic field $B_{\mathrm{T}}=1.8-2.3 \mathrm{~T}$ at major radius $R_{0}$. The $n=1 \mathrm{RMP}$ ELM suppression discharge on KSTAR can be reproduced at a lower electron density regime (.e.g., Greenwald density fraction $\sim 0.4$ ) with a plasma shape having elongation $\kappa \sim 1.71$, upper triangularity $\delta_{\text {up }} \sim 0.37$, and upper triangularity $\delta_{\text {low }} \sim 0.85$.

Radial profile reconstruction. Core ion temperature is measured by charge exchange recombination system ${ }^{51}$ for Carbon (6+) impurities at outboard mid-plane. Core electron temperature is measured by the Thomson Scattering ${ }^{52}$ and Electron Cyclotron emission ${ }^{53}$ system. Core electron density is measured by the Thomson Scattering and Two-color interferometry system ${ }^{54}$. To obtain well-resolved profiles, the data are averaged over $100 \mathrm{~ms}$. The pedestal height is obtained from hyperbolic tangent fits with edge profiles. The equilibria from EFIT $\operatorname{code}^{55}$ is used for the radial profile mapping and fitting. 
Kinetic equilibria reconstruction. Kinetic equilibria are reconstructed for the plasma stability analysis. This equilibrium is calculated from the magnetic reconstruction using EFIT code with the pressure profile (summation of thermal pressure profile from radial profile reconstruction and fast ion pressure from NUBEAM code ${ }^{56}$ ) and current density profile (core current from motional Stark effect diagnostics ${ }^{57}$ and edge current using NUBEAM, Ohmic and Sauter current models ${ }^{58}$ ) as a constraint. An iteration scheme is employed to update the thermal profiles, NUBEAM results, and edge current calculation with new kinetic equilibrium.

Pedestal stability calculation. The pedestal stability (or ELM stability) limit is predicted using the EPED $1^{59}$ algorithm. The fixed-boundary equilibrium code, $\mathrm{CHEASE}^{60}$, is used for accurate equilibrium mapping, and the ideal MHD stability code, MISHKA $1^{61}$, is employed for ideal peeling-ballooning ${ }^{3}$ stability calculation. The linear initial value solver is used to calculate the most unstable mode. All other required parameters are taken from the reconstructed radial profiles and plasma equilibrium.

Ideal plasma response calculation. The perturbed radial fields $\left(\delta B_{\mathrm{r}}\right)$ from an ideal plasma response by RMP are calculated using IPEC code ${ }^{62}$ and given magnetic equilibria and $I_{\text {RMP. The }}$ core and edge responses are derived through radially averaging $\delta B_{\mathrm{r}}$ at $\psi_{\mathrm{N}}=0-0.9$ and $0.9-1.0$, respectively. The thresholds of $\delta B_{\mathrm{r}}$ for RMP-induced ELM suppression and disruption are obtained from neighboring experiments. The disruption thresholds in $I_{\mathrm{RMP}}$ are equivalent to the $\delta B_{\mathrm{r}}$ thresholds based on the plasma response calculation.

Plasma fluctuation measurements. In this work, edge $T_{\mathrm{e}}$ and $n_{\mathrm{e}}$ fluctuations $\left(k_{\perp} \rho<1\right)$ are measured from electron emission image spectroscopy (ECEI) ${ }^{63}$ and beam emission spectroscopy $(\mathrm{BES})^{64}$, respectively. Magnetic field perturbations are captured by the Mirnov coil signal (MC) ${ }^{65}$. The spectrogram of measured fluctuation is derived using Fourier transform. Coherence of electron density and temperature fluctuation is calculated from bi-spectrum analysis with two radially adjacent channels in ECEI and BES, respectively. The ELM peaks and core modes are statistically removed from integrating the amplitude of coherent fluctuations in all channels.

Gyro-kinetic simulation. The gyrokinetic code, $\mathrm{CGYRO}^{66}$, is used in the linear analysis of 
micro-instabilities. The linear initial value solver is employed to find the unstable mode in the target radial point with wavelength $k_{\mathrm{y}} \rho_{\mathrm{s}}=0.1-1.5$. This simulation is based on a flux-tube approach with a full gyro-kinetic description for both electron and ion channels. The reconstructed radial profiles and kinetic equilibrium described above are included for the accurate modeling. This calculation is performed at $\psi_{\mathrm{N}}=0.96$, where the changes of experimental fluctuations are robust.

\section{ACKNOWLEDGMENTS}

The authors would like to thanks KSTAR team. This material was supported by the U.S. Department of Energy, under Awards DE-SC0020372. This research was also supported by R\&D Program of "KSTAR Experimental Collaboration and Fusion Plasma Research(EN2021-12)" through the Korea Institute of Fusion Energy(KFE) funded by the Government funds.

\section{AUTHOR CONTRIBUTIONS}

S.K. and R.S. led the experimental demonstration and analysis. R.S. and S.K. develop the adaptive controller. E.K. conceived the original idea of adaptive control. A.O.N. analyzed the micro instability with Gyro-kinetic code. S.H., S.Y., J.W. and Y.M. participated in all the experimental procedures and support the analysis. R.N. and Y.I. discussed the critical physics picture of ion-scale turbulence and other transports at the pedestal. J.P. and Y.S. discussed the role of RMP response, stability and transport analysis of pedestal region. C.Y conducted the interpretive transport analysis using ASTRA. J.L. and J.K. analyzed the measured edge fluctuation in electron channel. S.K. wrote the main manuscript text and A.O.N. and all authors reviewed it.

\section{ADDITIONAL INFORMATION}

Competing financial interests: The authors declare no competing financial interests. 


\section{REFERENCES}

${ }^{1}$ F. Wagner, G. Fussmann, T. Grave, M. Keilhacker, M. Kornherr, K. Lackner, K. McCormick, E. R. Müller, A. Stäbler, G. Becker, K. Bernhardi, U. Ditte, A. Eberhagen, O. Gehre, J. Gernhardt, G. v. Gierke, E. Glock, O. Gruber, G. Haas, M. Hesse, G. Janeschitz, F. Karger, S. Kissel, O. Klüber, G. Lisitano, H. M. Mayer, D. Meisel, V. Mertens, H. Murmann, W. Poschenrieder, H. Rapp, H. Röhr, F. Ryter, F. Schneider, G. Siller, P. Smeulders, F. Söldner, E. Speth, K. H. Steuer, Z. Szymanski, and O. Vollmer, en"Development of an Edge Transport Barrier at the H-Mode Transition of ASDEX," Physical Review Letters 53, 1453-1456 (1984).

${ }^{2}$ A. Sips, J. Schweinzer, T. Luce, S. Wolfe, H. Urano, J. Hobirk, S. Ide, E. Joffrin, C. Kessel, S. Kim, P. Lomas, I. Nunes, T. Pütterich, F. Rimini, W. Solomon, J. Stober, F. Turco, P. de Vries, JET Contributors, The ASDEX Upgrade team, The DIII-D team, The C-Mod team, The JT-60U team, and ITPA-IOS TG members and experts, en"Assessment of the baseline scenario at $q 95$ 3 for ITER," Nuclear Fusion 58, 126010 (2018).

${ }^{3}$ J. W. Connor, R. J. Hastie, H. R. Wilson, and R. L. Miller, en“Magnetohydrodynamic stability of tokamak edge plasmas," Physics of Plasmas 5, 2687-2700 (1998).

${ }^{4}$ A. Loarte, G. Huijsmans, S. Futatani, L. Baylor, T. Evans, D. M. Orlov, O. Schmitz, M. Becoulet, P. Cahyna, Y. Gribov, A. Kavin, A. Sashala Naik, D. Campbell, T. Casper, E. Daly, H. Frerichs, A. Kischner, R. Laengner, S. Lisgo, R. Pitts, G. Saibene, and A. Wingen, en“Progress on the application of ELM control schemes to ITER scenarios from the non-active phase to DT operation,” Nuclear Fusion 54, 033007 (2014).

${ }^{5}$ J. Gunn, S. Carpentier-Chouchana, F. Escourbiac, T. Hirai, S. Panayotis, R. Pitts, Y. Corre, R. Dejarnac, M. Firdaouss, M. Kočan, M. Komm, A. Kukushkin, P. Languille, M. Missirlian, W. Zhao, and G. Zhong, en“Surface heat loads on the ITER divertor vertical targets," Nuclear Fusion 57, 046025 (2017).

${ }^{6}$ T. E. Evans, R. A. Moyer, P. R. Thomas, J. G. Watkins, T. H. Osborne, J. A. Boedo, E. J. Doyle, M. E. Fenstermacher, K. H. Finken, R. J. Groebner, M. Groth, J. H. Harris, R. J. L. Haye, C. J. Lasnier, S. Masuzaki, N. Ohyabu, D. G. Pretty, T. L. Rhodes, H. Reimerdes, D. L. Rudakov, M. J. Schaffer, G. Wang, and L. Zeng, "Suppression of Large Edge-Localized Modes in HighConfinement DIII-D Plasmas with a Stochastic Magnetic Boundary," Physical Review Letters 92 (2004), 10.1103/physrevlett.92.235003, publisher: American Physical Society (APS). 
${ }^{7}$ W. Suttrop, T. Eich, J. C. Fuchs, S. Günter, A. Janzer, A. Herrmann, A. Kallenbach, P. T. Lang, T. Lunt, M. Maraschek, R. M. McDermott, A. Mlynek, T. Pütterich, M. Rott, T. Vierle, E. Wolfrum, Q. Yu, I. Zammuto, and H. Zohm, en“First Observation of Edge Localized Modes Mitigation with Resonant and Nonresonant Magnetic Perturbations in ASDEX Upgrade," Physical Review Letters 106, 225004 (2011).

${ }^{8}$ Y. M. Jeon, J.-K. Park, S. W. Yoon, W. H. Ko, S. G. Lee, K. D. Lee, G. S. Yun, Y. U. Nam, W. C. Kim, J.-G. Kwak, K. S. Lee, H. K. Kim, and H. L. Yang, "Suppression of Edge Localized Modes in High-Confinement KSTAR Plasmas by Nonaxisymmetric Magnetic Perturbations,” Physical Review Letters 109 (2012), 10.1103/physrevlett.109.035004, publisher: American Physical Society (APS).

${ }^{9}$ Y. Sun, Y. Liang, Y. \. e. Liu, S. Gu, X. Yang, W. Guo, T. Shi, M. Jia, L. Wang, B. Lyu, C. Zhou, A. Liu, Q. Zang, H. Liu, N. Chu, H. I. e. Wang, T. Zhang, J. Qian, L. Xu, K. He, D. Chen, B. Shen, X. Gong, X. Ji, S. Wang, M. Qi, Y. Song, Q. Yuan, Z. Sheng, G. Gao, P. Fu, and B. Wan, "Nonlinear Transition from Mitigation to Suppression of the Edge Localized Mode with Resonant Magnetic Perturbations in the EAST Tokamak,” Physical Review Letters 117 (2016), 10.1103/physrevlett.117.115001, publisher: American Physical Society (APS).

${ }^{10}$ M. E. Fenstermacher, T. E. Evans, T. H. Osborne, M. J. Schaffer, M. P. Aldan, J. S. deGrassie, P. Gohil, I. Joseph, R. A. Moyer, P. B. Snyder, R. J. Groebner, M. Jakubowski, A. W. Leonard, O. Schmitz, and the DIII-D Team, en"Effect of island overlap on edge localized mode suppression by resonant magnetic perturbations in DIII-D," Physics of Plasmas 15, 056122 (2008).

${ }^{11}$ F. L. Waelbroeck, I. Joseph, E. Nardon, M. Bécoulet, and R. Fitzpatrick, "Role of singular layers in the plasma response to resonant magnetic perturbations," Nuclear Fusion 52, 074004 (2012), publisher: IOP Publishing.

${ }^{12}$ Q. M. Hu, R. Nazikian, B. A. Grierson, N. C. Logan, J.-K. Park, C. Paz-Soldan, and Q. Yu, “The density dependence of edge-localized-mode suppression and pump-out by resonant magnetic perturbations in the DIII-D tokamak," Physics of Plasmas 26, 120702 (2019), publisher: AIP Publishing.

${ }^{13}$ R. Fitzpatrick, "Theory of edge localized mode suppression by static resonant magnetic perturbations in the DIII-D tokamak," Physics of Plasmas 27, 042506 (2020), publisher: AIP Publishing.

${ }^{14}$ Y. Liu, C. Paz-Soldan, L. Li, and Y. Sun, en"Role of 3D neoclassical particle flux in density pump-out during ELM control by RMP in DIII-D,” Nuclear Fusion 60, 036018 (2020). 
${ }^{15}$ V. Rozhansky, P. Molchanov, E. Kaveeva, S. Voskoboynikov, A. Kirk, E. Nardon, D. Coster, and M. Tendler, en"Modelling of the edge plasma of MAST in the presence of resonant magnetic perturbations," Nuclear Fusion 51, 083009 (2011).

${ }^{16} \mathrm{~S}$. Mordijck, R. A. Moyer, and G. R. McKee, en“'Changes in density fluctuations as a result of resonant magnetic perturbations correlate with the density inverse scale length," Physics of Plasmas 19, 024504 (2012).

${ }^{17}$ G. McKee, Z. Yan, C. Holland, R. Buttery, T. Evans, R. Moyer, S. Mordijck, R. Nazikian, T. Rhodes, O. Schmitz, and M. Wade, en"Increase of turbulence and transport with resonant magnetic perturbations in ELM-suppressed plasmas on DIII-D," Nuclear Fusion 53, 113011 (2013).

${ }^{18}$ H. Müller, T. Lunt, W. Suttrop, T. Eich, R. Fischer, J. Fuchs, A. Herrmann, M. Kočan, P. de Marné, and E. Wolfrum, en"Modification of scrape-off layer transport and turbulence by non-axisymmetric magnetic perturbations in ASDEX Upgrade," Journal of Nuclear Materials 438, S64-S71 (2013).

${ }^{19}$ N. Vianello, C. Rea, M. Agostini, R. Cavazzana, G. Ciaccio, G. De Masi, E. Martines, A. Mazzi, B. Momo, G. Spizzo, P. Scarin, M. Spolaore, P. Zanca, M. Zuin, L. Carraro, P. Innocente, L. Marrelli, M. E. Puiatti, and D. Terranova, en"Magnetic perturbations as a viable tool for edge turbulence modification," Plasma Physics and Controlled Fusion 57, 014027 (2015).

${ }^{20}$ C. Rea, N. Vianello, M. Agostini, R. Cavazzana, G. De Masi, E. Martines, B. Momo, P. Scarin, S. Spagnolo, G. Spizzo, M. Spolaore, and M. Zuin, en“Comparative studies of electrostatic turbulence induced transport in presence of resonant magnetic perturbations in RFX-mod," Nuclear Fusion 55, 113021 (2015).

${ }^{21}$ L. Cui, R. Nazikian, B. Grierson, E. Belli, T. Evans, N. Logan, D. Orlov, S. Smith, G. Staebler, and P. Snyder, en“The energy confinement response of DIII-D plasmas to resonant magnetic perturbations," Nuclear Fusion 57, 116030 (2017).

${ }^{22}$ S. Liu, N. Yan, Y. Liang, H. Zhang, J. Xu, G. Xu, L. Wang, R. Chen, G. Hu, Y. Ye, Y. Sun, T. Shi, H. Wang, M. Wu, X. Wu, S. Gu, M. Jia, N. Chu, Q. Ma, Y. Wang, T. Zhang, X. Han, L. Chen, J. Liu, S. Xu, H. Wang, N. Zhao, W. Zhang, J. Qian, L. Zeng, L. Xu, S. Wang, H. Liu, Q. Zang, Y. Yu, L. Liao, X. Gong, and EAST, en"Edge turbulence characteristics and transport during the ELM mitigation with $n=1$ resonant magnetic perturbation on EAST," Nuclear Fusion 60, $082001(2020)$. 
${ }^{23}$ M. W. Jakubowski, T. E. Evans, M. E. Fenstermacher, M. Groth, C. J. Lasnier, A. W. Leonard, O. Schmitz, J. G. Watkins, T. Eich, W. Fundamenski, R. A. Moyer, R. C. Wolf, L. B. Baylor, J. A. Boedo, K. H. Burrell, H. Frerichs, J. S. deGrassie, P. Gohil, I. Joseph, S. Mordijck, M. Lehnen, C. C. Petty, R. I. Pinsker, D. Reiter, T. L. Rhodes, U. Samm, M. J. Schaffer, P. B. Snyder, H. Stoschus, T. Osborne, B. Unterberg, E. Unterberg, and W. P. West, "Overview of the results on divertor heat loads in RMP controlled H-mode plasmas on DIII-D," Nuclear Fusion 49, 095013 (2009), publisher: IOP Publishing.

${ }^{24}$ P. Snyder, K. Burrell, H. Wilson, M. Chu, M. Fenstermacher, A. Leonard, R. Moyer, T. Osborne, M. Umansky, W. West, and X. Xu, en"Stability and dynamics of the edge pedestal in the low collisionality regime: physics mechanisms for steady-state ELM-free operation,” Nuclear Fusion 47, 961-968 (2007).

${ }^{25}$ R. Nazikian, C. Paz-Soldan, J. \. e. Callen, J. I. e. deGrassie, D. Eldon, T. \. e. Evans, N. \. e. Ferraro, B. \. e. Grierson, R. \. e. Groebner, S. \. e. Haskey, C. \. e. Hegna, J. \. e. King, N. \. e. Logan, G. I. e. McKee, R. I. e. Moyer, M. Okabayashi, D. \. e. Orlov, T. \. e. Osborne, J.-K. Park, T. I. e. Rhodes, M. I. e. Shafer, P. I. e. Snyder, W. I. e. Solomon, E. I. e. Strait, and M. I. e. Wade, "Pedestal Bifurcation and Resonant Field Penetration at the Threshold of EdgeLocalized Mode Suppression in the DIII-D Tokamak," Physical Review Letters 114 (2015), 10.1103/physrevlett.114.105002, publisher: American Physical Society (APS).

${ }^{26}$ C. Paz-Soldan, R. Nazikian, S. I. e. Haskey, N. I. e. Logan, E. I. e. Strait, N. \. e. Ferraro, J. I. e. Hanson, J. I. e. King, M. I. e. Lanctot, R. I. e. Moyer, M. Okabayashi, J.-K. Park, M. I. e. Shafer, and B. I. e. Tobias, "Observation of a Multimode Plasma Response and its Relationship to Density Pumpout and Edge-Localized Mode Suppression,” Physical Review Letters 114 (2015), 10.1103/physrevlett.114.105001, publisher: American Physical Society (APS).

${ }^{27}$ G. Lee, J. Kim, S. Hwang, C. Chang, H. Chang, M. Cho, B. Choi, K. Kim, K. Cho, S. Cho, K. Choh, C. Choi, J. Choi, J. Choi, I. Choi, C. Do, T. Ha, J. Han, J. Hong, K. Hong, N. Hur, I. Hwang, K. Im, H. Jhang, Y. Jung, B. Kim, D. Kim, G. Kim, H. Kim, J. Kim, J. Kim, W. Kim, Y. Kim, K. Kwon, M. Kyum, B. Lee, D. Lee, H. Lee, J. Lee, S. Lee, H. Na, Y. Oh, J. Park, H. Ri, Y. Ryoo, K. Song, H. Yang, J. Yang, B. Yoo, S. Yoo, N. Yoon, S. Yoon, G. You, K. You, W. Choe, D.-I. Choi, S. Jeong, D. Lee, Y. Bae, H. Kang, G. Kim, I. Ko, W. Namkung, J. Oh, Y. Bae, Y. Cho, B. Hong, G. Hong, C. Hwang, S. In, M. Ju, H. Lee, B. Oh, B. Yoon, S. Baang, H. Choi, J. Hwang, M. Kim, Y. Kim, S. Lee, J. Yee, C. Yoon, K.-H. Chung, S. Hong, Y. Hwang, S. Kim, Y. Kim, K. Chung, J. Lim, D. Ha, S. Oh, K. Ryu, Q. Wang, T. Ko, J. Joo, S. Suh, C. Choi, 
J. Lee, Y. Lee, H. Shin, I. Song, J. Baek, I. Han, Y. Koh, P. Park, C. Ryu, J. Cho, D. Hwang, Y. Kim, J. Schmidt, H. Park, G. Neilson, W. Reiersen, R. Simmons, S. Bernabei, F. Dahlgren, L. Grisham, S. Jardin, C. Kessel, J. Manickam, S. Medley, N. Pomphrey, J. Sinnis, T. Brown, R. White, K. Young, J. Schultz, P. Wang, L. Sevier, M. Carter, P. Ryan, D. Swain, D. Hill, W. Nevins, and B. Braams, en"The KSTAR project: An advanced steady state superconducting tokamak experiment," Nuclear Fusion 40, 575-582 (2000).

${ }^{28}$ I. P. E. G. o. C. Transport, I. P. E. G. o. C. Database, and I. P. B. Editors, "Chapter 2: Plasma confinement and transport," Nuclear Fusion 39, 2175-2249 (1999).

${ }^{29}$ H. Zohm, en“On the Minimum Size of DEMO,” Fusion Science and Technology 58, 613-624 (2010).

${ }^{30}$ F. Laggner, D. Eldon, A. Nelson, C. Paz-Soldan, A. Bortolon, T. Evans, M. Fenstermacher, B. Grierson, Q. Hu, D. Humphreys, A. Hyatt, R. Nazikian, O. Meneghini, P. Snyder, E. Unterberg, E. Kolemen, and t. DIII-D team, en"Real-time pedestal optimization and ELM control with 3D fields and gas flows on DIII-D,” Nuclear Fusion 60, 076004 (2020).

${ }^{31}$ Y. In, J.-K. Park, Y. M. Jeon, J. Kim, G. Y. Park, J.-W. Ahn, A. Loarte, W. H. Ko, H. H. Lee, J. W. Yoo, J. W. Juhn, S. W. Yoon, and H. P. and, "Enhanced understanding of non-axisymmetric intrinsic and controlled field impacts in tokamaks," Nuclear Fusion 57, 116054 (2017), publisher: IOP Publishing.

${ }^{32}$ C. Sung, G. Wang, T. L. Rhodes, S. P. Smith, T. H. Osborne, M. Ono, G. R. McKee, Z. Yan, R. J. Groebner, E. M. Davis, L. Zeng, W. A. Peebles, and T. E. Evans, en“Increased electron temperature turbulence during suppression of edge localized mode by resonant magnetic perturbations in the DIII-D tokamak," Physics of Plasmas 24, 112305 (2017).

${ }^{33}$ M. Kim, J. Lee, W. H. Ko, S.-H. Hahn, Y. In, Y. M. Jeon, W. Suttrop, S. K. Kim, G. Y. Park, J.-W. Juhn, and J. H. Lee, en"Pedestal electron collisionality and toroidal rotation during ELM-crash suppression phase under $n=1$ RMP in KSTAR," Physics of Plasmas 27, 112501 (2020).

${ }^{34}$ D. Eldon, E. Kolemen, J. Barton, A. Briesemeister, D. Humphreys, A. Leonard, R. Maingi, M. Makowski, A. McLean, A. Moser, and P. Stangeby, en"Controlling marginally detached divertor plasmas," Nuclear Fusion 57, 066039 (2017).

${ }^{35}$ T. Osborne, G. Jackson, Z. Yan, R. Maingi, D. Mansfield, B. Grierson, C. Chrobak, A. McLean, S. Allen, D. Battaglia, A. Briesemeister, M. Fenstermacher, G. McKee, P. Snyder, and The DIII-D Team, "Enhanced H-mode pedestals with lithium injection in DIII-D," Nuclear Fusion 55, 063018 (2015). 
${ }^{36}$ Q. Hu, R. Nazikian, B. Grierson, N. Logan, D. Orlov, C. Paz-Soldan, and Q. Yu, en“Wide Operational Windows of Edge-Localized Mode Suppression by Resonant Magnetic Perturbations in the DIII-D Tokamak," Physical Review Letters 125, 045001 (2020).

${ }^{37}$ V. Rozhansky, E. Kaveeva, P. Molchanov, I. Veselova, S. Voskoboynikov, D. Coster, A. Kirk, S. Lisgo, and E. Nardon, en"Modification of the edge transport barrier by resonant magnetic perturbations," Nuclear Fusion 50, 034005 (2010).

${ }^{38}$ E. Viezzer, M. Cavedon, P. Cano-Megias, E. Fable, E. Wolfrum, D. J. Cruz-Zabala, P. David, R. Dux, R. Fischer, G. F. Harrer, F. M. Laggner, R. M. McDermott, U. Plank, T. Pütterich, M. Willensdorfer, and the ASDEX Upgrade Team, en"Dynamics of the pedestal transport during edge localized mode cycles at ASDEX Upgrade," Plasma Physics and Controlled Fusion 62, 024009 (2020).

${ }^{39}$ W. A. Houlberg, K. C. Shaing, S. P. Hirshman, and M. C. Zarnstorff, en"Bootstrap current and neoclassical transport in tokamaks of arbitrary collisionality and aspect ratio," Physics of Plasmas 4, 3230-3242 (1997).

${ }^{40}$ S. Mordijck, R. Moyer, N. Ferraro, M. Wade, and T. Osborne, en“The radial electric field as a measure for field penetration of resonant magnetic perturbations,” Nuclear Fusion 54, 082003 (2014).

${ }^{41}$ J. Lee, Y. M. Jeon, Y. In, G. Y. Park, G. S. Yun, W. Lee, M. Kim, J. H. Lee, W. H. Ko, and H. K. P. and, "Direct evidence of $\mathrm{E} \times \mathrm{B}$ flow changes at the onset of resonant magnetic perturbationdriven edge-localized mode crash suppression," Nuclear Fusion 59, 066033 (2019), publisher: IOP Publishing.

${ }^{42}$ J.-K. Park, Y. Jeon, Y. In, J.-W. Ahn, R. Nazikian, G. Park, J. Kim, H. Lee, W. Ko, H.-S. Kim, N. C. Logan, Z. Wang, E. A. Feibush, J. E. Menard, and M. C. Zarnstroff, “3D field phasespace control in tokamak plasmas," Nature Physics 14, 1223-1228 (2018), publisher: Springer Science and Business Media LLC.

${ }^{43}$ X. Chen, K. Burrell, T. Osborne, W. Solomon, K. Barada, A. Garofalo, R. Groebner, N. Luhmann, G. McKee, C. Muscatello, M. Ono, C. Petty, M. Porkolab, T. Rhodes, J. Rost, P. Snyder, G. Staebler, B. Tobias, Z. Yan, and the DIII-D Team, en“Stationary QH-mode plasmas with high and wide pedestal at low rotation on DIII-D,” Nuclear Fusion 57, 022007 (2017).

${ }^{44}$ R. Nazikian, C. Petty, A. Bortolon, X. Chen, D. Eldon, T. Evans, B. Grierson, N. Ferraro, S. Haskey, M. Knolker, C. Lasnier, N. Logan, R. Moyer, D. Orlov, T. Osborne, C. Paz-Soldan, F. Turco, H. Wang, and D. Weisberg, en“Grassy-ELM regime with edge resonant magnetic 
perturbations in fully noninductive plasmas in the DIII-D tokamak," Nuclear Fusion 58, 106010 (2018).

${ }^{45}$ J. Lee, G. S. Yun, M. J. Choi, J.-M. Kwon, Y.-M. Jeon, W. Lee, N. C. Luhmann, and H. K. Park, "Nonlinear Interaction of Edge-Localized Modes and Turbulent Eddies in Toroidal Plasma undern=1Magnetic Perturbation,” Physical Review Letters 117 (2016), 10.1103/physrevlett.117.075001, publisher: American Physical Society (APS).

${ }^{46} \mathrm{H}$. Biglari, P. H. Diamond, and P. W. Terry, "Influence of sheared poloidal rotation on edge turbulence," Physics of Fluids B: Plasma Physics 2, 1-4 (1990), publisher: AIP Publishing.

${ }^{47}$ T. S. Hahm and K. H. Burrell, "Flow shear induced fluctuation suppression in finite aspect ratio shaped tokamak plasma," Physics of Plasmas 2, 1648-1651 (1995), publisher: AIP Publishing.

${ }^{48}$ R. Hager, C. S. Chang, N. M. Ferraro, and R. Nazikian, en"Gyrokinetic understanding of the edge pedestal transport driven by resonant magnetic perturbations in a realistic divertor geometry," Physics of Plasmas 27, 062301 (2020), arXiv: 2003.07130.

${ }^{49}$ S. Taimourzadeh, L. Shi, Z. Lin, R. Nazikian, I. Holod, and D. Spong, en"Effects of RMPinduced changes of radial electric fields on microturbulence in DIII-D pedestal top," Nuclear Fusion 59, 046005 (2019).

${ }^{50}$ S. Yang, J.-K. Park, N. Logan, C. Zhu, Q. Hu, Y. Jeon, Y. In, W. Ko, S. Kim, Y. Lee, and Y. Na, "Localizing resonant magnetic perturbations for edge localized mode control in KSTAR," Nuclear Fusion 60, 096023 (2020).

${ }^{51}$ Won-Ha Ko, Seungtae Oh, and Myeun Kwon, en“KSTAR Charge Exchange Spectroscopy System," IEEE Transactions on Plasma Science 38, 996-1000 (2010).

${ }^{52}$ J. H. Lee, S. Oh, H. M. Wi, W. R. Lee, K. P. Kim, K. team, I. Yamada, K. Narihara, and K. Kawahata, en"Tangential Thomson scattering diagnostic for the KSTAR tokamak," Journal of Instrumentation 7, C02026-C02026 (2012).

${ }^{53}$ G. S. Yun, W. Lee, M. J. Choi, J. B. Kim, H. K. Park, C. W. Domier, B. Tobias, T. Liang, X. Kong, N. C. Luhmann, and A. J. H. Donné, en“Development of KSTAR ECE imaging system for measurement of temperature fluctuations and edge density fluctuations," Review of Scientific Instruments 81, 10D930 (2010).

${ }^{54}$ K. Lee, J.-W. Juhn, Y. Nam, Y. Kim, H. Wi, S. Kim, and Y.-c. Ghim, en“The design of two color interferometer system for the 3-dimensional analysis of plasma density evolution on KSTAR," Fusion Engineering and Design 113, 87-91 (2016). 
${ }^{55}$ L. Lao, H. St. John, R. Stambaugh, A. Kellman, and W. Pfeiffer, en“Reconstruction of current profile parameters and plasma shapes in tokamaks," Nuclear Fusion 25, 1611-1622 (1985).

${ }^{56}$ A. Pankin, D. McCune, R. Andre, G. Bateman, and A. Kritz, en“"The tokamak Monte Carlo fast ion module NUBEAM in the National Transport Code Collaboration library," Computer Physics Communications 159, 157-184 (2004).

${ }^{57}$ J. Chung, J. Ko, J. Howard, C. Michael, G. v. Nessi, A. Thorman, and M. F. M. D. Bock, "Motional Stark effect diagnostics for KSTAR," Journal of the Korean Physical Society 65, 1257-1260 (2014), publisher: Korean Physical Society.

${ }^{58}$ O. Sauter, C. Angioni, and Y. R. Lin-Liu, en“Neoclassical conductivity and bootstrap current formulas for general axisymmetric equilibria and arbitrary collisionality regime," Physics of Plasmas 6, 2834-2839 (1999).

${ }^{59}$ P. B. Snyder, R. J. Groebner, A. W. Leonard, T. H. Osborne, and H. R. Wilson, en“Development and validation of a predictive model for the pedestal height," Physics of Plasmas 16, 056118 (2009).

${ }^{60}$ H. Lütjens, A. Bondeson, and O. Sauter, "The CHEASE code for toroidal MHD equilibria," Computer Physics Communications 97, 219-260 (1996), publisher: Elsevier BV.

${ }^{61}$ A. Mikhailovskii, G. Huysmans, W. Kerner, and S. Sharapov, "Optimization of computational MHD normal-mode analysis for tokamaks," Plasma Physics Reports 23, 844-857 (1997).

${ }^{62}$ J.-k. Park, A. H. Boozer, and A. H. Glasser, en“Computation of three-dimensional tokamak and spherical torus equilibria," Physics of Plasmas 14, 052110 (2007).

${ }^{63}$ G. S. Yun, W. Lee, M. J. Choi, J. Lee, M. Kim, J. Leem, Y. Nam, G. H. Choe, H. K. Park, H. Park, D. S. Woo, K. W. Kim, C. W. Domier, N. C. Luhmann, N. Ito, A. Mase, and S. G. Lee, “Quasi 3D ECE imaging system for study of MHD instabilities in KSTAR,” Review of Scientific Instruments 85, 11D820 (2014), publisher: AIP Publishing.

${ }^{64}$ Y. U. Nam, S. Zoletnik, M. Lampert, Kovácsik, and H. M. Wi, en“Edge electron density profiles and fluctuations measured by two-dimensional beam emission spectroscopy in the KSTAR," Review of Scientific Instruments 85, 11E434 (2014).

${ }^{65}$ J. G. Bak, S. G. Lee, D. Son, and the KSTAR Project Team, en“Performance of the magnetic sensor and the integrator for the KSTAR magnetic diagnostics," Review of Scientific Instruments 75, 4305-4307 (2004).

${ }^{66}$ J. Candy, E. Belli, and R. Bravenec, en“A high-accuracy Eulerian gyrokinetic solver for collisional plasmas," Journal of Computational Physics 324, 73-93 (2016). 